\title{
A network pharmacology study on analgesic mechanism of Yuanhu-Baizhi herb pair
}

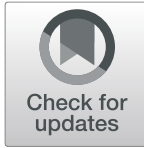

Bobin $\mathrm{Mi}^{1}$, Qiushi $\mathrm{Li}^{2}$, Tong $\mathrm{Li}^{3}$, Jessica Marshall ${ }^{4}$ and Jiayang Sai ${ }^{1,3^{*}}$

\begin{abstract}
Background: Millions of people are suffering from chronic pain conditions, such as headache, arthritis, cancer. Apart from western medicines, traditional Chinese medicines are also well accepted for pain management, especially in Asian countries. Yuanhu-Baizhi herb pair (YB) is a typical herb pair applied to the treatment of stomach pain, hypochondriac pain, headache, and dysmenorrhea, due to its effects on analgesia and sedation. This study is to identify potentially active compounds and the underlying mechanisms of $Y B$ in the treatment of pain.

Methods: Compounds in YB were collected from 3 online databases and then screened by bioavailability and drug likeness parameters. Swiss target prediction was applied to obtain targets information of the active compounds. Pain-related genes were conducted for Gene ontology (GO) and Kyoto Encyclopedia of Genes and Genomes (KEGG) analysis. Protein-protein interaction (PPI) networks of the genes were constructed using Cytoscape software. In addition, the hub genes were screened using maximal clique centrality (MCC) algorithm.

Results: In total, 31 compounds from Yuanhu were screened out with 35 putative target genes, while 26 compounds in Baizhi with 43 target genes were discovered. Hence, 78 potential target genes of YB were selected for further study. After overlap analysis of the 78 genes of YB and 2408 pain-associated genes, we finally achieved 34 YB-pain target genes, as well as 10 hub genes and 23 core compounds. Go enrichment and KEGG pathway analysis indicated that YB had a strong integration with neuro system, which might significantly contribute to antinociceptive effect.

Conclusion: Our data provide deep understanding of the pharmacological mechanisms of YB in attenuating pain. The discovery shed new light on the development of active compounds of $Y B$ for the treatment of pain.
\end{abstract}

Keywords: Rhizoma Corydalis, Radix angelicae dahuricae, Pain, Analgesic, Network pharmacology

\section{Background}

Pain is a common syndrome related to various diseases, such as cancer, fracture, etc. It is estimated that $20 \%$ of individuals around the worldwide have some degree of chronic pain [1]. Adequate pain assessment and rational management are essential to improve the quality

\footnotetext{
*Correspondence: saijiayang90@126.com

'Department of Orthopaedics, Union Hospital, Tongji Medical College, Huazhong University of Science and Technology, Wuhan 430022, China

${ }^{3}$ Department of oncology, The Third Affiliated Hospital, Beijing University of Chinese Medicine, Beijing 100029, China

Full list of author information is available at the end of the article
}

of life in this population. Although stepwise escalation of analgesic therapy (paracetamol, non-steroidal antiinflammatory drugs, mild to strong opioids) according to the World Health Organization's three-step pain ladder works well for majority patients suffering from pain [2], the prevailing adverse effect still exist among patients after using the primary drugs. Thus, adjuvants are always recommended for the management of nociceptive pain to maximize nonopioids and minimize long-term opioid use in patients who may live for decades with a chronic pain syndrome. 
Nowadays, accumulative studies provide substantial evidence that traditional Chinese medical therapy, including herbs, formulas, etc. has an additive effect when used in combination with opioids or may be used as single agent treatment for pain relief [3, 4].Yuanhu-Baizhi herb pair (YB), consisting of Corydalis yanhusuo W. T. Wang (Yuanhu in Chinese) and Angelica dahurica (Fisch.ex Hoffm.) Benth. et Hook. f. (Baizhi in Chinese), is well-known for its analgesic effect [5]. Diverse forms of YB, such as capsules, pills, and oral solution, have been developed and widely used in the treatment of stomachache, headache and dysmenorrhea. In addition, previous studies have proved pain alleviating effects of YB [5-7]. Considering the widely clinical use of Yuanhu and Baizhi on pain management, the active compounds and potential targets of $\mathrm{YB}$ on analgesia has yet to be discovered.

Traditional Chinese medicine (TCM) network pharmacology is a preferred method to study herb-compoundsdiseases-targets because of its capacity of describing complex interactions between drugs and biological systems and the "multi-component, multi-target, and multipathway" characteristics of TCM. Therefore, in the present study, we are committed to screen the active compounds in YB that may modulate pain-related genes. Besides, the underlying mechanism of YB-induced pain relief was investigated.

\section{Methods}

\section{Active compounds and targets}

All of the chemical monomer compounds in YB were retrieved from The Encyclopedia of Traditional Chinese Medicine (ETCM, http://www.nrc.ac.cn:9090/ETCM/), Traditional Chinese Medicine Information Database (TCMID, http://bidd.nus.edu.sg/group/TCMsite/), and TCMGeneDIT (http://tcm.lifescience.ntu.edu.tw/). ETCM includes comprehensive information for the commonly used herbs and formulas of TCM, as well as their ingredients. TCMID is a systemic platform designed to identify informative materials on all aspects of TCM including formulation, herbal composition, chemical composition, etc. TCMGeneDIT serves as a database system providing association information about TCMs, genes, diseases, TCM effects and TCM ingredients automatically mined from vast amount of biomedical literature.

\section{Pharmacokinetic predictions}

Canonical Simplified Molecular-Input Line-Entry System (SMILES) of compounds were collected from three databases, including Swiss ADME (http://www.swissadme. ch/), Pubchem (https://pubchem.ncbi.nlm.nih.gov/), and ChEMBL (https://www.ebi.ac.uk/chembl/). Then five important pharmacology-related properties were obtained from Swiss ADME by accurate searching canonical
SMILES, including MW, ALogP, Hbond donor count, and Hbond acceptor count, Rotation bond count. Those properties were applied to the drug likeness evaluation based on Lipinski's rule of five (RO5) [8]. Systemic evaluation of ADME (absorption, distribution, metabolism, excretion, toxicity) was carried out by ADMETlab (http://admet.scbdd.com/), among which, F (30\% Bioavailability) and drug likeness (DL) were collected for further screening. Then, compounds with F $(30 \%) \geq 30 \%$ and $\mathrm{DL} \geq 30 \%$ were identified as active compounds.

\section{Targets prediction}

The potential targets of active compounds of YB are predicted by Swiss ADME and collected using a probability $\geq 30 \%$. The pain-associated target genes were obtained from six databases, including DISGeNET [9], drugbank [10], GeneCards [11], The Online Mendelian Inheritance in Man database (OMIM) [12], Therapeutic Target Database (TTD) [13], and The Human Protein Atlas (THPA) [14]. The species was set to Homo sapiens. Venn diagram was drawn for overlap analysis to obtain potential pain-associated target genes of active compounds.

\section{Network construction}

Protein-Protein Interaction (PPI) data were obtained from the Search Tool for the Retrieval of Interacting Genes (STRING) database and a confidence score of $>$ 0.4 was selected to construct PPI network [15]. The networks were generated using Cytoscape (version 3.7.1) to further illustrate scientific interpretation of the complicated relationships among genes.

\section{Enrichment analysis}

Gene Ontology (GO) Enrichment and Kyoto Encyclopedia of Genes and Genomes (KEGG) Pathway Enrichment Analysis were carried out using DAVID tool (https://david.ncifcrf.gov/) [16].

\section{Results \\ Drug likeness and bioavailability analysis for compounds in YB}

In this study, we obtained 47 compounds in Yuanhu (Corydalis yanhusuo W. T. Wang) and 84 compounds in Baizhi (Angelica dahurica (Fisch.ex Hoffm.) Benth. et Hook. f., Supplementary Excel.1). Then we selected compounds using descriptors retrieved from Swiss ADME, results in 43 compounds out of 47 in Yuanhu and 77 out of 84 in Baizhi, respectively. The statistic results of their drug-like property descriptors were listed in Table 1. Further screening was carried out based on $\mathrm{F}$ $(30 \%) \geq 30 \%$ and $\mathrm{DL} \geq 30 \%$ collected from ADMETlab. Finally, we obtained 41 compounds in Yuanhu and 64 compounds in Baizhi (Supplementary Excel. 2). 
Table 1 Drug-like property descriptors of compounds in YB

\begin{tabular}{lllll}
\hline Herbs & Descriptors & Median & Mean & Std \\
\hline Yanhu & MW & 341.40 & 332.74 & 53.60 \\
& a_acc & 5.00 & 4.86 & 0.95 \\
& a_don & 1.00 & 0.67 & 0.84 \\
& logP(o/w) & 2.79 & 2.59 & 0.88 \\
Baizhi & MW & 204.35 & 208.61 & 73.31 \\
& a_acc & 2.00 & 2.44 & 2.30 \\
& a_don & 0.00 & 0.66 & 0.95 \\
& logP(o/w) & 3.24 & 3.00 & 1.62 \\
\hline
\end{tabular}

\section{YB compound-target network}

Swiss Target Prediction was applied to identify target genes of active compounds. After screening using a probability $\geq 30 \%, 31$ active compounds from Yuanhu with 35 putative target genes, and 26 active compounds in Baizhi with 43 putative target genes were identified in the results. The interaction between active compounds and genes were constructed by Cytoscape. (Fig. 1a, b).

As shown in Fig. 1, the network showed that compounds which connected to the most target genes were tetrahydropalmatine, codeine, lirioferine etc., indicating that these compounds might play a major role in Yuanhu, while cetylic acid, Neobyakangelicol etc., might be the critical compounds in Baizhi. In addition, we listed the compound-candidate target network parameters in Table 2.

\section{Overlap analysis between YB targets and pain associated targets}

Six databases were mined including TTD, DISGeNET, DrugBank, Genecards, OMIM, and The Human Protein Atlas to screen the pain-related genes. In total, 2408
Table 2 YB compound-candidate target network parameters

\begin{tabular}{lll}
\hline Herbs & Network parameter & Values \\
\hline Yanhu & Number of nodes & 64 \\
& Network heterogeneity & 0.830 \\
& Average number of neighbors & 5.969 \\
& Characteristic path length & 3.088 \\
& Shortest paths & $3784(93 \%)$ \\
& Network centralization & 0.246 \\
Baizhi & Number of nodes & 68 \\
& Network heterogeneity & 0.931 \\
& Average number of neighbors & 4.824 \\
& Characteristic path length & 2.473 \\
& Shortest paths & $1930(42 \%)$ \\
& Network centralization & 0.156 \\
\hline
\end{tabular}

pain-related genes were obtained. After overlapping analysis, 34 frequently affected therapeutic target genes for pain in active compounds of YB were discovered (Fig. 2). We also created a PPI network for all the 34 genes with Cytoscape (settings: Homo sapiens and confidence $>0.4$ ) and 10 hub genes with plugcluster Cytohubba by maximal clique centrality (MCC) method were screened. To be more specific, the 34 targets were as follows: CYP2D6, KCNA1, HTR7, DRD3, ADRA2A, OPRK1, CHRM1, CHRNB2, ADRA1A, HTR1A, DRD4, HTR1D, HTR2A, SLC6A3, ADRA2C, SLC6A4, PTPN11, HTR2C, KCNA2, MAPT, DRD2, ADRA1B, SIGMAR1, PPARA, CNR2, HTR2B, HTR1B, ADRA2B, CHRNA4, HTR6, OPRD1, KCNA5, OPRM1, and PTGS1 (Fig. 3a). Among which, the top 10 hub genes were DRD4, HTR1A, DRD2, OPRD1, DRD3, OPRM1, OPRK1, HTR1B, ADRA2C, and ADRA2A (Fig. 3b, c). The PPI network was rebuilt with top 8 hub genes and related active

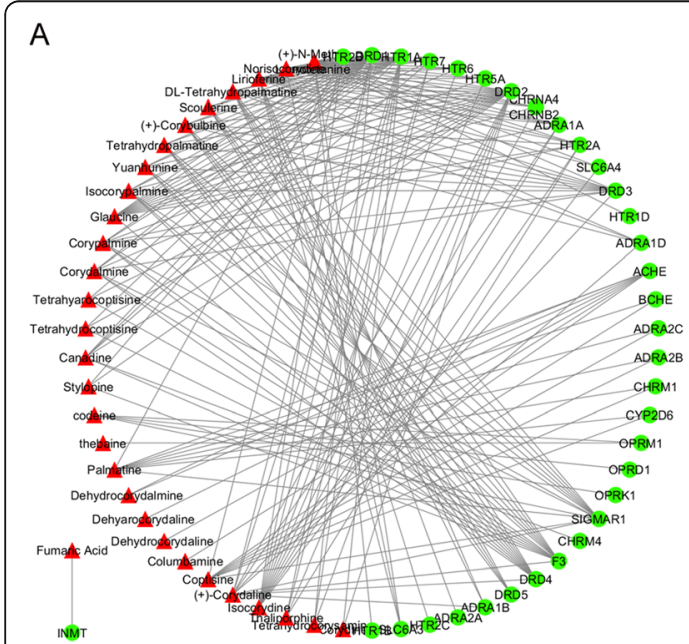

B

Fig. 1 Yuanhu and Baizhi compound-target network. a Yuanhu compound-target network. b Bai zhi compound-target network. Red represents compounds and green represents the targets of compounds 


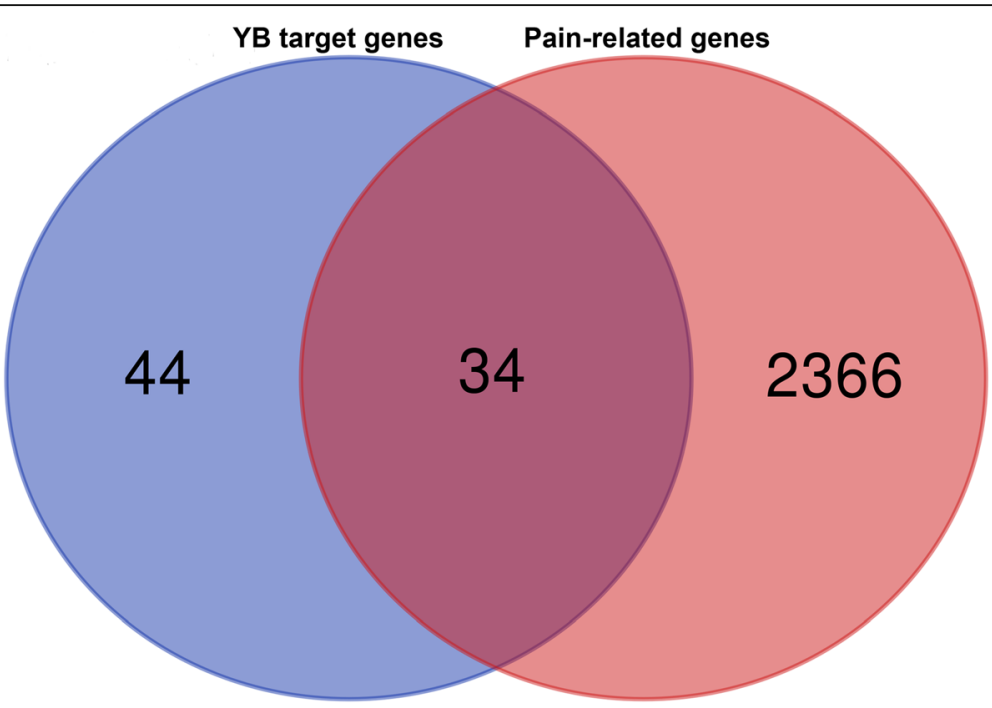

Fig. 2 Venn Diagram for the overlap analysis of YB tageted genes and pain related genes

compounds of $\mathrm{YB}$, resulting in 23 core compounds, which may be active therapeutic compounds related with pain relief in YB (Fig. 4).

\section{GO enrichment and KEGG enrichment}

To further explore the multiple mechanisms of YB as a therapy drug against pain, GO enrichment analysis of 34 target genes shared by YB and pain was performed using DAVID bioinformatics resources. The top 10 significantly enriched terms including biological process (BP), molecular function (MF), and cellular component (CC) are presented $(p$-value $<0.05)$ in Fig. $5 \mathrm{a}-\mathrm{c}$. The top 10 MF pathways included: drug binding, neurotransmitter receptor activity, G-protein coupled serotonin receptor activity, serotonin binding, dopamine binding, alpha2- adrenergic receptor activity, dopamine neurotransmitter receptor activity, dopamine neurotransmitter receptor activity, epinephrine binding, opioid receptor activity, which are all classical pathways involved in pain inducing and relieving. $\mathrm{BP}$ and $\mathrm{CC}$ terms analysis indicate that $\mathrm{YB}$ are mainly involved in chemical synaptic transmission, $G$ protein coupled receptor signaling, and locomotory behavior. What's more, YB are strongly related to the integral component of plasma membrane, cell junction, and dendrite.

The KEGG enrichment analysis of 34 target genes was performed to explore the potential biological pathways. We obtained 9 pathways in total which belong to several categories, including neuroactive ligand receptor interaction, serotonergic synapse, calcium signaling pathway,

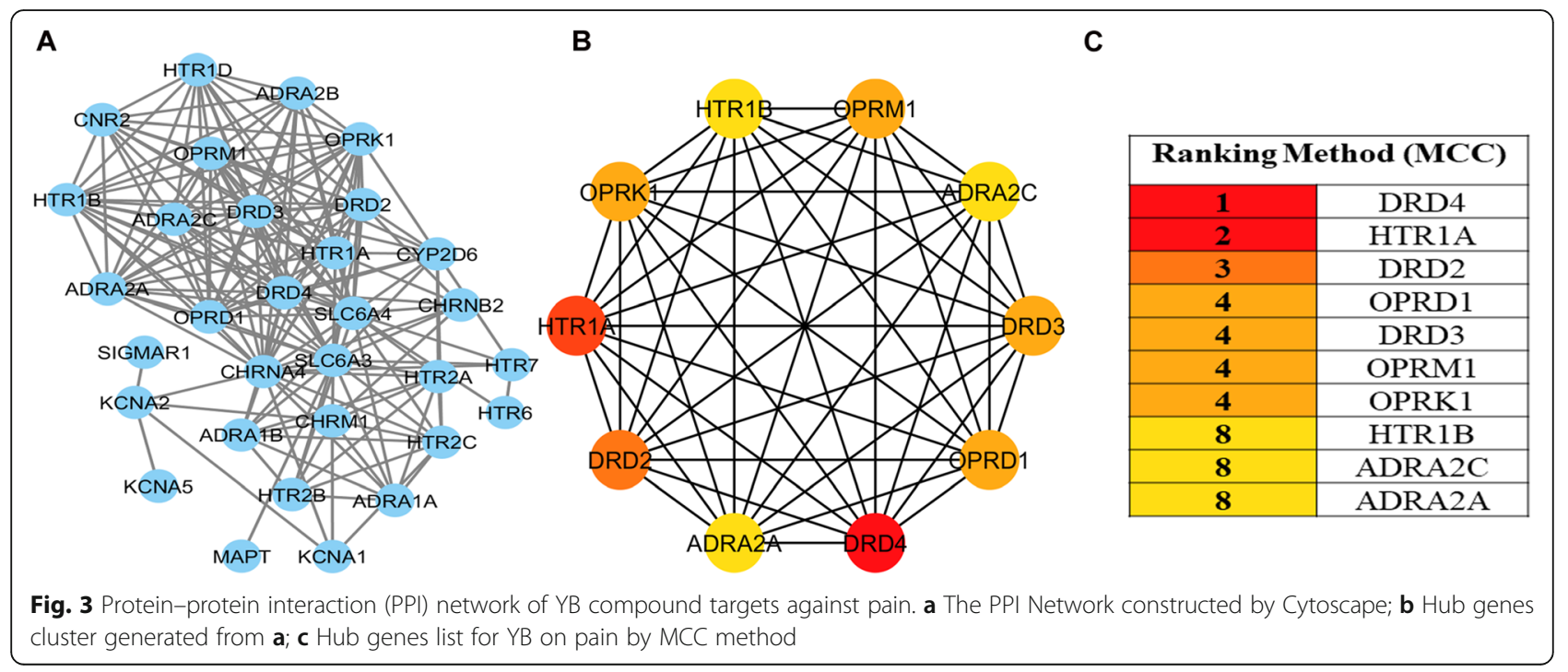




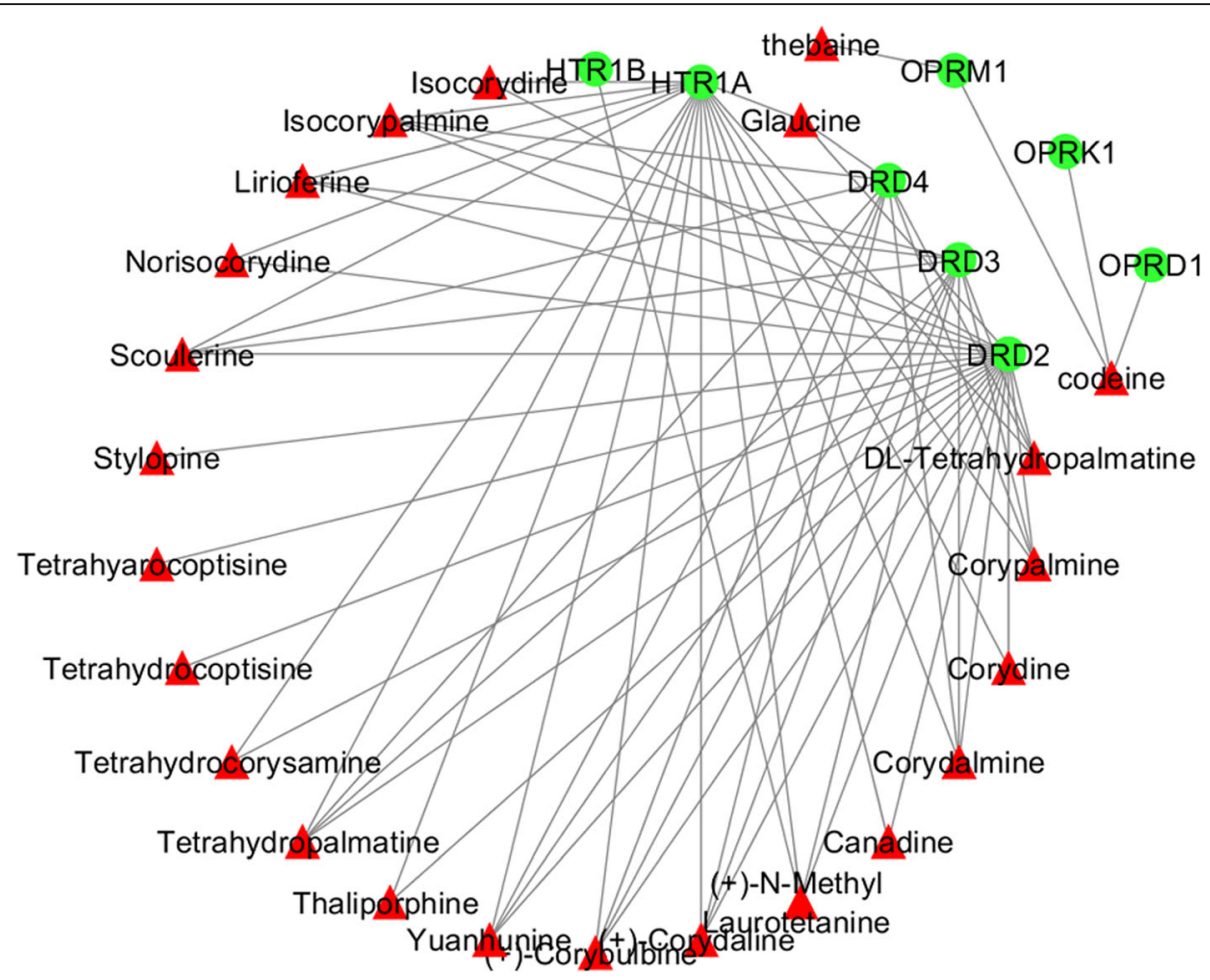

Fig. 4 The network for 8 YB-related pain target genes and their interactive YB compounds. Green nodes represent pain-related targeted genes, and red nodes represent $\mathrm{YB}$ active compounds

cAMP signaling pathway, dopaminergic synapse, etc. (Fig. 5d).

\section{Discussion}

As Traditional Chinese Medicine (TCM) has been widely accepted around the world, there are still several problems to be addressed, among which, the active components and target genes have always been the issue and key point for TCM modernization [17, 18]. While existing methods mainly concerned the indicative ingredients and their potential pharmacological effects, network pharmacology study emerged as a more powerful method to identify active compounds and target genes due to multi-component and multi-target mode of TCM [19]. Le et al. reported that 7 alkaloids from Yuanhu and 8 coumarins from Baizhi were selected as active compounds by LC-MS/MS method [5]. In the present study, three databases were mined, resulting in 47 compounds in Yuanhu and 84 compounds in Baizhi. In total, 131 compounds were obtained from $\mathrm{YB}$, which provided more compounds for further analysis. Normally, $\mathrm{OB} \geq$ $30 \%$ and $\mathrm{DL} \geq 0.18$ are considered chemically suitable for drug development, they are used as the included criteria of bioactive compounds in most literatures [20]. In the current study, we calculated drug likeness probability and $\mathrm{F}$ (30\% Bioavailability) probability of active compounds of YB using ADMETlab. Bioavailability, which is referred to as the degree and rate at which the active compound is absorbed by the systemic circulation, is calculated by Random forests (RF) method with accuracy of 0.669 and AUC score of 0.715 by fivefold cross validation. DL, which is defined as a complex balance of molecular properties and structure features, is closely related to bioactivity and bioavailability. DL is evaluated using one well-performed classification model with classification accuracy of 0.800 and AUC score of 0.867 by external test set [21]. An oral bioactivity of $\geq 20 \%$ is considered acceptable to identify compounds with accepted oral bioavailability. In this study, $\mathrm{F} \geq 30 \%$ and $\mathrm{DL} \geq 30 \%$ were used as screening criteria, which was stricter and more rigorous [22-24].

As shown in PPI network of Yuanhu, the most frequently targeted genes are ACHE, HTR1A, DRD2, HTR2A, HTR7, DRD1, HTR2B, DRD1, HTR7, HTR6, HTR5A, OPRM1, F3. Amongst them, the target genes in the neuro system accounted most, indicating that Yuanhu plays an important role in neuroprotection. While the top frequently targeted genes in Baizhi are CA12, CA9, CA7, PTGS1, DYRK1A, BACE1, BACE2, MBNL1. Among which, CAs, BACE, are involved in mechanism of neuronal protection against ischemia. PTGS1, known as Cyclooxygenase 1 (COX-1), are involved in osteogenic differentiation [25], cancer prevention and therapy [26], etc. And DYRK1A, MBNL1 are 


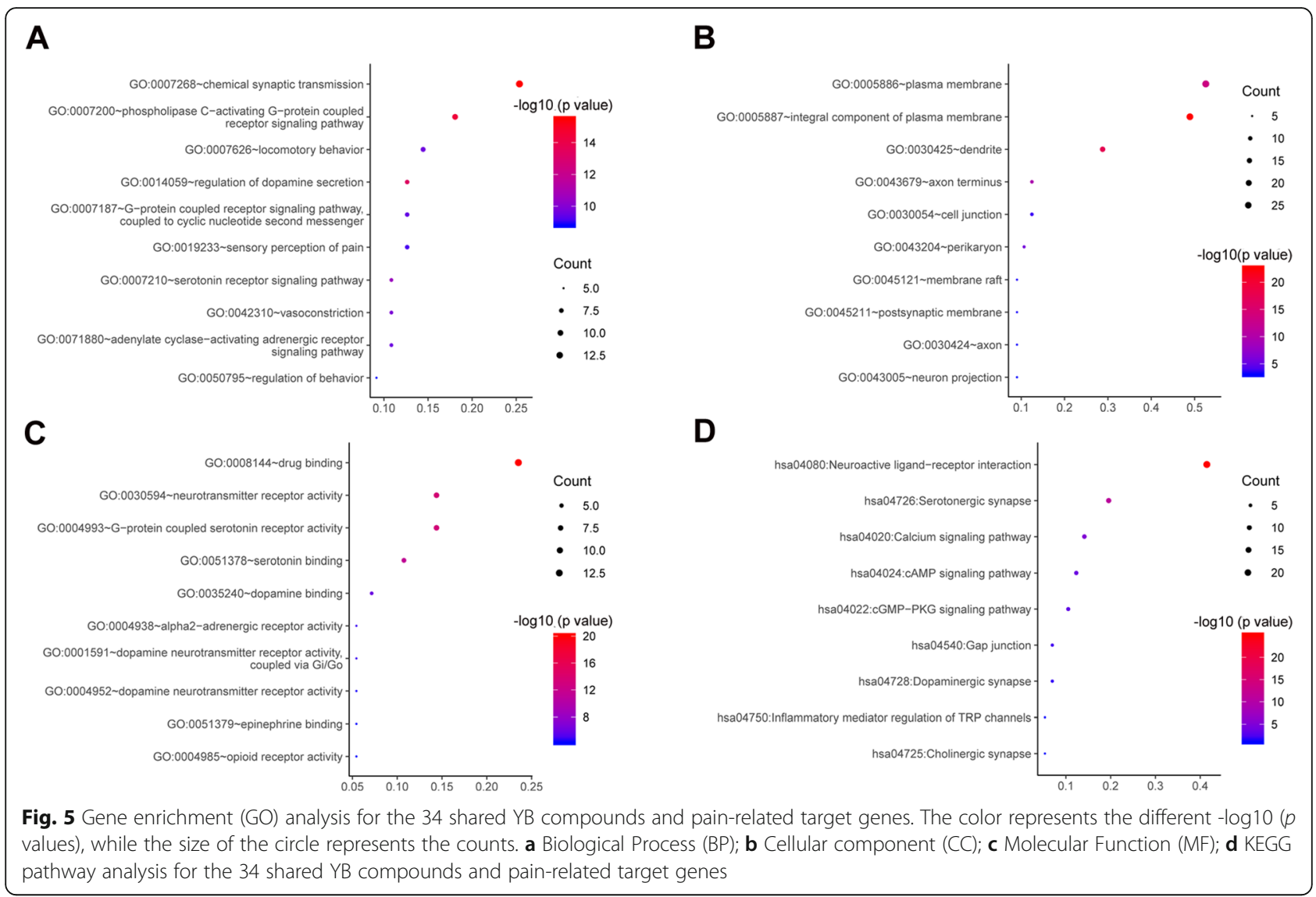

most involved in immune system [27], which suggest that Baizhi may be active in neuro-immune system. Dysfunctional pain is thought to arise from altered processing of nociceptive information in the central nervous system [28], and primary sensory neurons are involved in both acute and chronic pain [29], we deduct that YB may interact with neuro system to exert analgesia effect.

The PPI network also presents top 8 hub genes and its related 23 core compounds in YB. The top 8 genes actually belong to 3 families, dopamine receptors, 5hydroxytryptamine (serotonin) receptors, and opioid receptors, which all are typical pain related genes, indicating that $\mathrm{YB}$ is definitely a pain-relieving candidate. Besides, the 34 genes targeted by YB contribute unevenly to the mechanism of analgesia effect. Take CYP2D6 for instance. CYP2D6 is the most frequently addressed candidate gene in the literature on pain, which is involved in the biological activation of codeine into morphine and tramadol into O-desmethyltramadol [30]. Based on the extensive evidence for this gene, the availability of guidelines for healthcare professionals, and the fact that active metabolites of codeine and tramadol are formed by this enzyme, this is a highly suitable biomarker for improving pain therapy in the clinic [31]. The sigma-1 receptor (SIGMAR1) is reported to be involved in pain modulation especially under pre-sensitized conditions [32], and related to CNS inflammation [33]. KCNA1 were found to be associated with membership in the mild pain class [34], endogenous Kcna2 antisense RNA was suggested as a therapeutic target for the treatment of neuropathic pain [35], while KCNA5 is mainly involved in drug uptake [36]. The alpha-2-adrenergic receptors targeted by $\mathrm{YB}$, including ADRA2A, ADRA1A, ADRA2C, ADRA1B, ADRA2B, are involved in regulating the release of neurotransmitter molecules from sympathetic nerves and from adrenergic neurons in the central nervous system. SLC6A4, a member of neurotransmitter symporter family, can be targeted by psychomotor stimulants, such as amphetamines and cocaine and can terminate the action of serotonin and recycles it in a sodium-dependent manner. While SLC6A3 is a dopamine transporter. In addition, MAPT, CNR2 CHRM1, CHRNB2, CHRNA4 are all neuro-related proteins. While PTPN11, PPARA, PTGS1 have been shown to be more involved in cell proliferation. All the lines of evidence suggest that $\mathrm{YB}$ function as an analgesia through multi targets, amongst which, neuroprotection may account the most. This indicates that YB may be more suitable for chronic pain than acute ones, which is more related to inflammation. 
The 23 compounds are as follows: Stylopine, Tetrahydropalmatine, Canadine, (+)-Corybulbine, (+)-Corydaline, Corydalmine, Corydine, Corypalmine, Glaucine, Isocorydine, Isocorypalmine, Lirioferine, (+)-N-Methyl Laurotetanine, Norisocorydine, Scoulerine, Tetrahydrocoptisine, Tetrahydrocorysamine, Thaliporphine, Yuanhunine, DL-Tetrahydropalmatine, codeine, thebaine. Among which, Glaucine, tetrahydropalmatine, canadine, corydaline, and tetrahydrocoptisine were determined relatively high in Rhizoma corydalis extract by HPLC-TOF/MS and they were absorbed into blood quickly [37]. Codeine is a wellknown natural plant alkaloid commonly used to treat mild-to-moderate pain and cough [38]. Thebaine (paramorphine), chemically similar to codeine and morphine, is not used therapeutically but often converted to codeine for use. Dehydrocorybulbine (DHCB), has been identified as a dopamine receptor antagonist, exhibiting high to moderate binding affinities to sigma 1 and 2 receptors, serotonin 5-HT7 receptor, and histamine $\mathrm{H} 2$ receptors [39]. While (R)glaucine appears to act as a positive allosteric modulator at the 5-HT2A receptor [40]. Levotetrahydropalmatine (L-THP) and Isocorypalmine exerted analgesic effects by agonism D1R and antagonism D2R [41, 42]. Levo-corydalmine attenuates vincristine-induced neuropathic pain by regulating the CXCL1/CXCR2 signaling pathway [43].

We also discovered several compounds in $\mathrm{YB}$, which have not been reported to be related with analgesia in previous studies. However, they did have important functions in diverse diseases. For instance, tetrahydrocoptisine was reported to exert neuropsychopharmacological property in 1976 [44]. Thaliporphine, along with reperfusion therapy conferred cardioprotection via activation of opioid receptor [45]. Stylopine, which also exits in Chelidonium majus L. (Papaveraceae), is tested to have anti inflammation activity [46]. While Lirioferine and (+)-N-Methyl Laurotetanine were shown as good candidate with antileishmanial activity [47]. Isocorydine can selectively inhibit human cancer stem cells, which have an important role in the development of chemoresistance [48]. Scoulerine is a potent antimitotic compound and it merits further investigation as an anticancer drug [49]. These lines of evidence demonstrate that YB may act on multiple targets to play their pharmacological roles on pain. What's more, little reports were found about Canadine, (+)-Corybulbine, (+)-Corydaline, Corydine, Corypalmine, Norisocorydine, Tetrahydrocorysamine, Yuanhunine, which may act as potential compounds for pain relief. It will be interesting to develop the potential active compounds as new anti-pain drugs.

\section{Conclusion}

In summary, we found more potential compounds in YB involved in intrinsic control of pain besides the wellknown ones, which still need further study to be verified about their specific roles in YB on pain. In addition, some crucial target genes, besides the already reported ones tested in animal experiments, such as CYP2D6, SIGMAR1, KCNA1, alpha-2-adrenergic receptors, SLC6A3, MAPT, CNR2 CHRM1, CHRNB2, CHRNA4, all may play significant roles in YB for attenuating pain.

\section{Supplementary information}

Supplementary information accompanies this paper at https://doi.org/10. 1186/s12906-020-03078-0.

\section{Additional file 1.}

Additional file 2.

\section{Acknowledgements}

The authors would like to express their gratitude to the Chinse Scholar Counsel for their support.

\section{Authors' contributions}

JS conceived the research theme and supervised the implementation. BM and QL designed the method and performed the experiments. JS wrote the manuscript. TL and JM revised the language. All authors read and approved the final manuscript.

Funding

Not applicable.

\section{Availability of data and materials}

The datasets used and/or analyzed in the current study are available from the corresponding author on request.

\section{Ethics approval and consent to participate}

All experiments were reviewed and approved by the Committee of Ethics on Human Experiments (S2243) and were carried out under the Guidelines for Animal Experiments at the Huazhong University of Science and Technology. A written signed informed consent was provided by all individual participants included in the study.

\section{Consent for publication}

Not applicable.

\section{Competing interests}

The authors declare that they have no competing interests.

\section{Author details}

${ }^{1}$ Department of Orthopaedics, Union Hospital, Tongji Medical College, Huazhong University of Science and Technology, Wuhan 430022, China. ${ }^{2}$ Department of Cardiology, Beijing Chaoyang Integrative Medicine Emergency Medical Center, Beijing 100029, China. ${ }^{3}$ Department of oncology, The Third Affiliated Hospital, Beijing University of Chinese Medicine, Beijing 100029, China. ${ }^{4}$ Department of Surgery, Brigham and Women's Hospital, Harvard Medical School, Boston, 02115, Boston, MA 02115, USA.

Received: 20 March 2020 Accepted: 13 September 2020

Published online: 18 September 2020

\section{References}

1. Varrassi G, Yeam CT, Rekatsina M, Pergolizzi JV, Zis P, Paladini A. The Expanding Role of the COX Inhibitor/Opioid Receptor Agonist Combination in the Management of Pain. Drugs. 2020;1-11. [Epub ahead of print].

2. Scarborough BM, Smith CB. Optimal pain management for patients with cancer in the modern era. CA Cancer J Clin. 2018;68(3):182-96. 
3. Xu L, Lao LX, Ge A, Yu S, Li J, Mansky PJ. Chinese herbal medicine for cancer pain. Integr Cancer Ther. 2007;6(3):208-34.

4. Yuan QL, Guo TM, Liu L, Sun F, Zhang YG. Traditional Chinese medicine for neck pain and low back pain: a systematic review and meta-analysis. PLoS One. 2015;10(2):e0117146.

5. Le J, Lin Z, Song L, Wang H, Hong Z. LC-MS/MS combined with in vivo microdialysis sampling from conscious rat striatum for simultaneous determination of active constituents of Yuanhu- Baizhi herb pair and endogenous neurotransmitters: application to pharmacokinetic and pharmacodynamic study. J Pharm Biomed Anal. 2019;176:112807.

6. Gu Y, Huang J, Guo H, Song X, Li J, Shi Y, et al. A randomized controlled study for Yuanhu Zhitong dropping pills in the treatment of knee osteoarthritis. Medicine (Baltimore). 2020;99(24):e20666.

7. Wang P, Li K, Tao Y, Li D, Zhang Y, Xu H, et al. TCM-ADMEpred: a novel strategy for poly-pharmacokinetics prediction of traditional Chinese medicine based on single constituent pharmacokinetics, structural similarity, and mathematical modeling. J Ethnopharmacol. 2019;236:277-87.

8. Lipinski CA. Lead- and drug-like compounds: the rule-of-five revolution. Drug Discov Today Technol. 2004;1 (4):337-41.

9. Pinero J, Bravo A, Queralt-Rosinach N, Gutierrez-Sacristan A, Deu-Pons J, Centeno E, et al. DisGeNET: a comprehensive platform integrating information on human disease-associated genes and variants. Nucleic Acids Res. 2017:45(D1):D833-D9.

10. Barneh F, Jafari M, Mirzaie M. Updates on drug-target network; facilitating polypharmacology and data integration by growth of DrugBank database. Brief Bioinform. 2016;17(6):1070-80.

11. Safran M, Dalah I, Alexander J, Rosen N, Iny Stein T, Shmoish M, et al. GeneCards Version 3: the human gene integrator. Database (Oxford). 2010;2010:baq020.

12. Hamosh A, Scott AF, Amberger JS, Bocchini CA, McKusick VA. Online Mendelian inheritance in man (OMIM), a knowledgebase of human genes and genetic disorders. Nucleic Acids Res. 2005;33(Database issue):D514-7.

13. Wang Y, Zhang S, Li F, Zhou Y, Zhang Y, Wang Z, et al. Therapeutic target database 2020: enriched resource for facilitating research and early development of targeted therapeutics. Nucleic Acids Res. 2020;48(D1):D1031-D41.

14. Uhlen M, Oksvold P, Fagerberg L, Lundberg E, Jonasson K, Forsberg M, et al. Towards a knowledge-based human protein atlas. Nat Biotechnol. 2010; 28(12):1248-50.

15. Szklarczyk D, Morris JH, Cook H, Kuhn M, Wyder S, Simonovic M, et al. The STRING database in 2017: quality-controlled protein-protein association networks, made broadly accessible. Nucleic Acids Res. 2017;45(D1):D362-D8.

16. Dennis G Jr, Sherman BT, Hosack DA, Yang J, Gao W, Lane HC, et al. DAVID: database for annotation, visualization, and integrated discovery. Genome Biol. 2003;4(5):3.

17. Li S, Zhang B. Traditional Chinese medicine network pharmacology: theory, methodology and application. Chin J Nat Med. 2013;11(2):110-20.

18. Zhang R, Zhu X, Bai H, Ning K. Network pharmacology databases for traditional Chinese medicine: review and assessment. Front Pharmacol. 2019;10:123.

19. Wang L, Li Z, Shao Q, Li X, Ai N, Zhao X, et al. Dissecting active ingredients of Chinese medicine by content-weighted ingredient-target network. Mol BioSyst. 2014;10(7):1905-11.

20. Liu H, Wang J, Zhou W, Wang Y, Yang L. Systems approaches and polypharmacology for drug discovery from herbal medicines: an example using licorice. J Ethnopharmacol. 2013;146(3):773-93.

21. Dong J, Wang NN, Yao ZJ, Zhang L, Cheng Y, Ouyang D, et al. ADMETlab: a platform for systematic ADMET evaluation based on a comprehensively collected ADMET database. J Cheminform. 2018;10(1):29.

22. Tian S, Li Y, Wang J, Zhang J, Hou T. ADME evaluation in drug discovery. 9. Prediction of oral bioavailability in humans based on molecular properties and structural fingerprints. Mol Pharm. 2011;8(3):841-51.

23. Ma CY, Yang SY, Zhang H, Xiang ML, Huang Q, Wei YQ. Prediction models of human plasma protein binding rate and oral bioavailability derived by using GA-CG-SVM method. J Pharm Biomed Anal. 2008;47(4-5):677-82.

24. Veber DF, Johnson SR, Cheng HY, Smith BR, Ward KW, Kopple KD. Molecular properties that influence the oral bioavailability of drug candidates. J Med Chem. 2002;45(12):2615-23

25. Wang Y, Liu Y, Zhang M, Lv L, Zhang X, Zhang P, et al. Inhibition of PTGS1 promotes osteogenic differentiation of adipose-derived stem cells by suppressing NF-kB signaling. Stem Cell Res Ther. 2019;10(1):57.

26. Benelli R, Vene R, Ferrari N. Prostaglandin-endoperoxide synthase 2 (cyclooxygenase-2), a complex target for colorectal cancer prevention and therapy. Transl Res. 2018;196:42-61.
27. Valencic E, Piscianz E, Sirchia F, Tommasini A, Faletra F, Todaro F, et al. Tregs and Th17 lymphocytes in human DYRK1A haploinsufficiency. Immunol Lett. 2019;214:52-4.

28. Ruscheweyh R, Wilder-Smith O, Drdla R, Liu XG, Sandkuhler J. Long-term potentiation in spinal nociceptive pathways as a novel target for pain therapy. Mol Pain. 2011;7:20.

29. Bennett DL, Clark AJ, Huang J, Waxman SG, Dib-Hajj SD. The role of voltagegated sodium channels in pain signaling. Physiol Rev. 2019;99(2):1079-151.

30. Mercadante S. Opioid metabolism and clinical aspects. Eur J Pharmacol. 2015;769:71-8.

31. Matic M, de Wildt SN, Tibboel D, van Schaik RHN. Analgesia and opioids: a Pharmacogenetics shortlist for implementation in clinical practice. Clin Chem. 2017;63(7):1204-13.

32. Sachau J, Bruckmueller H, Gierthmuhlen J, Magerl W, Kaehler M, Haenisch S, et al. SIGMA-1 receptor gene variants affect the somatosensory phenotype in neuropathic pain patients. J Pain. 2019;20(2):201-14.

33. Wheeler MA, Jaronen M, Covacu R, Zandee SEJ, Scalisi G, Rothhammer V, et al. Environmental control of astrocyte pathogenic activities in CNS inflammation. Cell. 2019;176(3):581-96 e18.

34. Langford DJ, Paul SM, West CM, Dunn LB, Levine JD, Kober KM, et al. Variations in potassium channel genes are associated with distinct trajectories of persistent breast pain after breast cancer surgery. Pain. 2015;156(3):371-80.

35. Zhao X, Tang Z, Zhang H, Atianjoh FE, Zhao JY, Liang L, et al. A long noncoding RNA contributes to neuropathic pain by silencing Kcna2 in primary afferent neurons. Nat Neurosci. 2013;16(8):1024-31.

36. Yang T, McBride BF, Leake BF, Kim RB, Roden DM. Modulation of drug block of the cardiac potassium channel KCNA5 by the drug transporters OCTN1 and MDR1. Br J Pharmacol. 2010;161(5):1023-33.

37. Hong Z, Zhao L, Wang X, Le J, Jia J, Chai Y, et al. High-performance liquid chromatography-time-of-flight mass spectrometry with adjustment of fragmentor voltages for rapid identification of alkaloids in rat plasma after oral administration of rhizoma Corydalis extracts. J Sep Sci. 2012;35(13):1690-6.

38. Celic I, Bach-Rojecky L, Mercep I, Soldo A, Petrak AK, Bucan A. Resolving issues about efficacy and safety of low-dose codeine in combination analgesic drugs: a systematic review. Pain Ther. 2020;9(1):171-94.

39. Wang $L$, Zhang $Y$, Wang $C$, Zhang $X$, Wang Z, Liang $X$, et al. A natural product with high affinity to sigma and 5 -HT7 receptors as novel therapeutic drug for negative and cognitive symptoms of schizophrenia. Neurochem Res. 2019:44(11):2536-45.

40. Heng HL, Chee CF, Thy CK, Tee JT, Chin SP, Herr DR, et al. In vitro functional evaluation of isolaureline, dicentrine and glaucine enantiomers at 5-HT2 and alpha1 receptors. Chem Biol Drug Des. 2019;93(2):132-8.

41. Wang JB, Mantsch JR. L-tetrahydropalamatine: a potential new medication for the treatment of cocaine addiction. Future Med Chem. 2012;4(2):177-86.

42. Xu W, Wang Y, Ma Z, Chiu YT, Huang P, Rasakham K, et al. L-isocorypalmine reduces behavioral sensitization and rewarding effects of cocaine in mice by acting on dopamine receptors. Drug Alcohol Depend. 2013;133(2):693-703.

43. Zhou L, Hu Y, Li C, Yan Y, Ao L, Yu B, et al. Levo-corydalmine alleviates vincristineinduced neuropathic pain in mice by inhibiting an NF-kappa B-dependent CXCL1/CXCR2 signaling pathway. Neuropharmacology. 2018;135:34-47.

44. Bhattacharya SK, Pandey VB, Ray AB, Dasgupta B. Neuropsychopharmacological studies with (--)-tetrahydrocoptisine. Short communication. Arzneimittelforschung. 1976;26(12):2187.

45. Chang WL, Lee SS, Su MJ. Attenuation of post-ischemia reperfusion injury by thaliporphine and morphine in rat hearts. J Biomed Sci. 2005;12(4):611-9.

46. Jang SI, Kim BH, Lee WY, An SJ, Choi HG, Jeon BH, et al. Stylopine from Chelidonium majus inhibits LPS-induced inflammatory mediators in RAW 264.7 cells. Arch Pharm Res. 2004;27(9):923-9.

47. Correa JE, Rios CH, del Rosario CA, Romero LI, Ortega-Barria E, Coley PD, et al. Minor alkaloids from Guatteria dumetorum with antileishmanial activity. Planta Med. 2006;72(3):270-2.

48. Pan JX, Chen G, Li JJ, Zhu QD, Li JJ, Chen ZJ, et al. Isocorydine suppresses doxorubicin-induced epithelial-mesenchymal transition via inhibition of ERK signaling pathways in hepatocellular carcinoma. Am J Cancer Res. 2018;8(1):154-64.

49. Habartova K, Havelek R, Seifrtova M, Kralovec K, Cahlikova L, Chlebek J, et al. Scoulerine affects microtubule structure, inhibits proliferation, arrests cell cycle and thus culminates in the apoptotic death of cancer cells. Sci Rep. 2018:8(1):4829.

\section{Publisher's Note}

Springer Nature remains neutral with regard to jurisdictional claims in published maps and institutional affiliations. 